\title{
INCORPORATION OF SUPERPOSITION INTO THE PROGRAM POISSON*
}

S. Caspi, M. Helm, and L.J. Laslett

\author{
Lawrence Berkeley Laboratory \\ University of California \\ Berkeley, California 94720
}

\author{
January 1985
}

\footnotetext{
* This work was supported by the Director, Office of Energy Research, Office of High Energy and Nuclear Physics, High Energy Physics Division, U.S. Dept. of Energy, under Contract No. DE-AC03-76SF00098.
} 


\section{INCORPORATION OF SUPERPOSITION INTO THE PROGRAM POISSON*}

S. Caspi, M. Helm and L. J. Laslett

Lawrence Berkeley Laboratory

University of California

Berkeley, California 94720

\section{Introduction}

In a previous series of reports $(1,2,3)$, we have outlined and incorpo:ated a mathematical analysis of a circular and elliptical boundary into the program POISSON thus making it possible to calculate with good accuracy the vector potential (and hence the field) of problems which have little or no symmetry.

The analysis was based on the assumption that no sources are present outside the boundary introduced. As will be shown, this condition can be waived by incorporating superposition into the relaxation process in a way that solutions to electromagnetic problems which are affected by an outside field (such as the earth magnetic field) can be obtained. Such solutions are also possible in the area of hydrodynamics. Similarities in the physical laws that govern electromagnetism and incompressible invicid hydrodynamics indicate that POISSON can be used as a tool to solve such problems. It is, however, the introduction of boundary condition and superposition into the relaxation process of POISSON that make such solutions practical.

We have introduced a combination of superposition and boundary condition into the relaxation process of POISSON in a manner that solutions can be obtained to electromagnetic problems placed in a background field as well as

* This work was supported by the Office of Energy Research, Office of High Energy and Nuclear Physics, High Energy Physics Division, U.S. Dept. of Energy, under Contract No. DE-AC03-76SF00098. 
two-dimensional hydrodynamic problems involving potential flow and circulation. We have named the new program FOIL. In simple terms, the total potential, reduced by such background contributions, is subjected to the type of boundary treatment that was employed previously when no external "sources" were present.

\section{Analysis}

FOIL calculates the vector potential $A_{j}$ of each mesh point on the boundary by updating it with vector potential values of points on the inner pseudo boundary. This has previously been expressed as:

$$
A_{i}^{\text {outer }}=\sum_{j=1}^{N} E_{i j} A_{j}^{\text {inner }}
$$

The matrix $E_{i j}$ which takes care of the geometry and symmetry, is based on the assumption that no sources exist outside the boundary. If we now assume that outside sources are present and their vector potential function $A_{A}^{\text {Source is }}$ known, we can define a superposed vector potential $A$ Super on the inner boundary as:

$$
A_{i}^{\text {Super-inner }}=A_{i}^{i n n e r}-A_{i}^{\text {Source-inner }}
$$

Note that $A_{i}^{\text {Source-inner }}$ is known and $A_{i}^{\text {inner }}$ has been calculated by the

relaxation process.

The next step is to update the values of the vector potential on the outer boundary according to:

$$
A_{i}^{\text {outer }}=\sum_{j=1}^{N} E_{i j} A_{j}^{\text {Super-inner }}+A_{i}^{\text {Source-outer }}
$$


Once the outer boundary has been updated, the relaxation process is permitted to continue relaxing the entire mesh before executing relations 2 and 3 once more. This process is to continue until convergence is obtained.

\section{Uniform Flow and Circulation}

The vector potential (stream function) for uniform flow, $U_{x}$ in the $x$ direction and $U_{y}$ in the $Y$ direction, and for the circulation $\Gamma$ is written as:

$$
A^{\text {Source }}=\left(U_{x} \sin \theta-U_{y} \cos \theta\right) r+\Gamma \ln r
$$

from which $U_{X}=\partial A / \partial Y$ and $U_{y}=-\partial A / \partial X$ (in analogy to $B=\nabla \times A \hat{e}_{z}$ in similar magnetostatic problems). Note that in the literature the value of $\Gamma$ is usually divided by $2 \pi$. This expression was introduced into FOIL (FOIL is a clone of POISSON with the exception of the inclusion of equations 2 and 3 ) and the following examples are used for demonstration.

\section{Uniform Flow over a Cylinder with Circulation}

In this example we have a cylinder of radius $r=4.0$, a flow velocity $U_{x}=1.0, U_{y}=0.0$ and a circulation $\Gamma=8.0$.

These values were chosen in such a way that the combination $\Gamma /(r U)=2$ coincides with the limiting case of a single stagnation point on the cylinder. This was intentionally done to achieve a simple visual effect of the stream line, Fig. 1. 


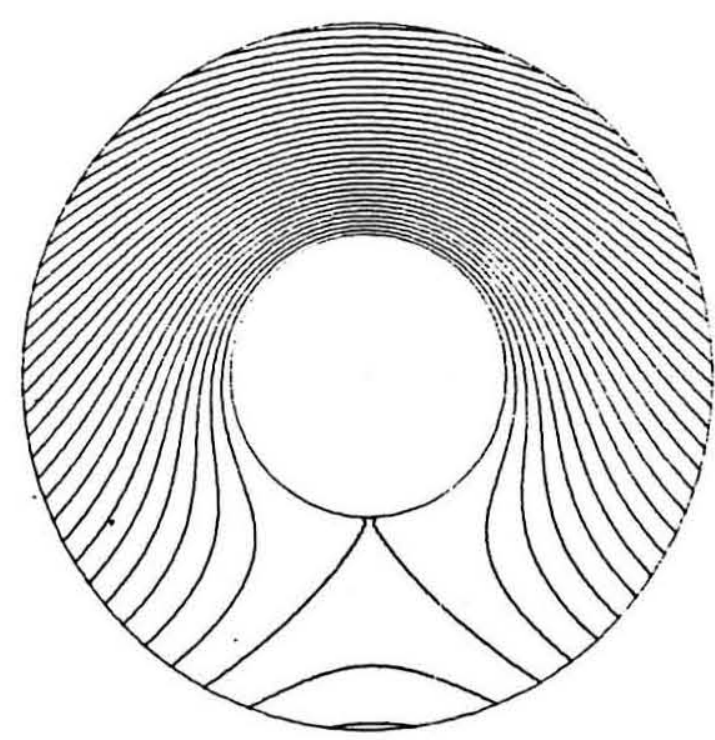

Fig. 1. Uniform flow over a cylinder with circulation, $\Gamma /(r U)=2$.

Uniform Flow Over an Airfoil

A more complete example is introduced by calculating the flow over a Kutta-Joukowski airfoil both analytically and by this method. We have an airfoil with a contour that corresponds to the complex transformation:

$\zeta=Z+\frac{c^{2}}{Z^{2}}$ of a displaced circle of radius $r=a$ centered at $z=x_{c}+i Y_{c}$ and passing through the point $Z=c$. (Appendix $A$ includes a short refreshment.) For such a body the circulation can be expressed as:

$$
\Gamma=2 a U \sin (\beta+\alpha)
$$

where $B=\arcsin \left(\frac{Y_{C}}{a}\right)$ and $\alpha$ is the angle between the wind velocity and the $x$ axis.

If we choose $\alpha=0.0, U_{X}=1.0, Y_{c}=0.30, X_{c}=-0.15$ and $c=1.0$ (resulting in a circle with $a=\sqrt{1.4125}$ and the appropriate value $\Gamma=0.6$ for the circulation), we arrive at the solution shown in Fig. 2. (See also Appendix B.) 


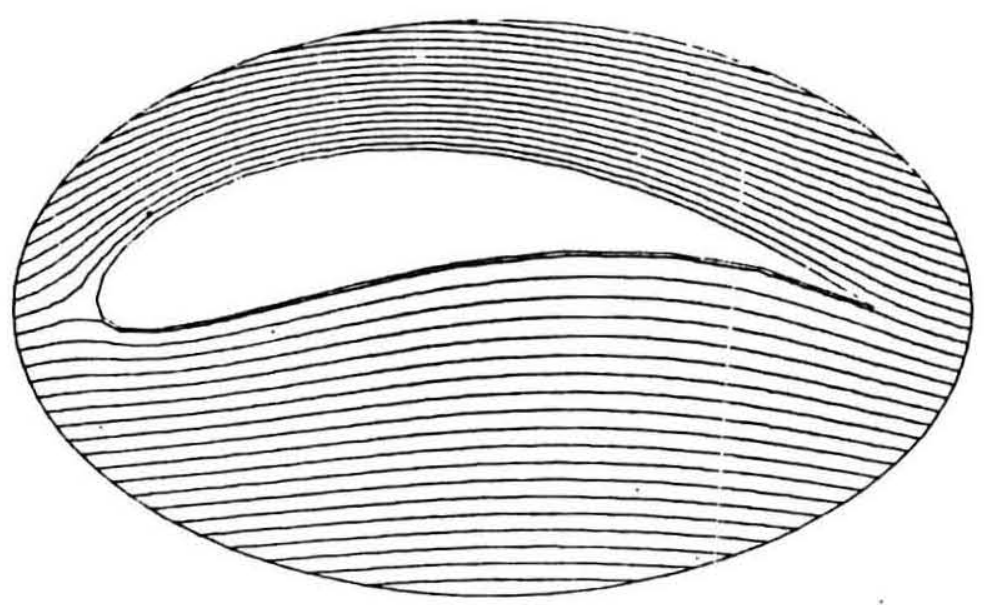

Fig. 2. Uniform flow over an airfoil, $\Gamma=0.6$.

Note that the stream line at the trailing edge appears continuous with the airfoil. Deviations from the theoretical value of $\Gamma$ result in stream line plots shown in Fig. $3 a, b, c$, with $\Gamma=0.6,0.5$, and 0.7 , respectively. The discontinuity in the streamline at the trailing edge of the airfoil is visual. This points out the way of estimating the approximate circulation for bodies of arbitrary shape.

As a final check we have calculated analytically the absolute values of the velocity at several points and checked them against numerical values. These are compared in Table 1. 


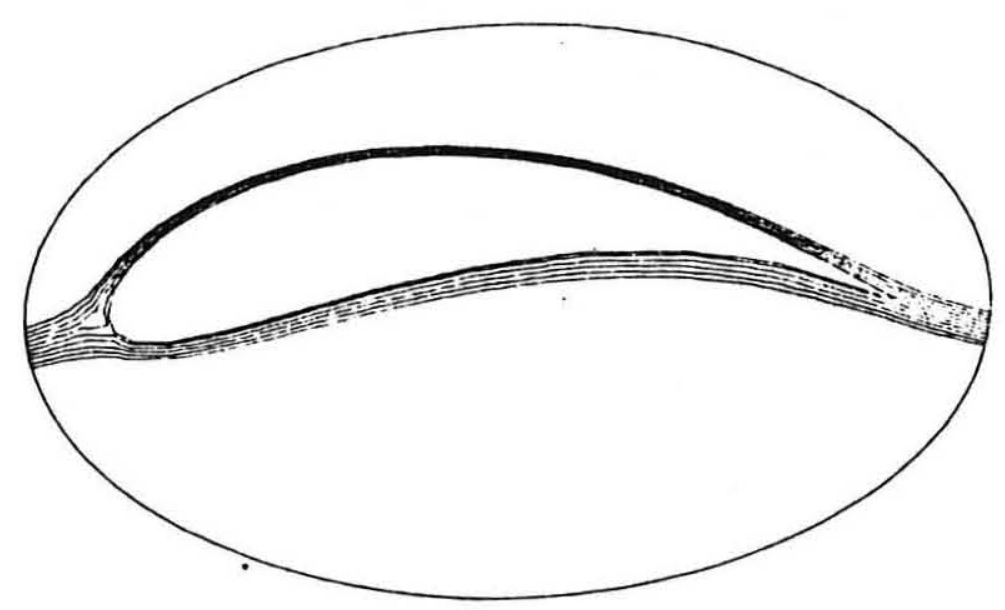

(a)

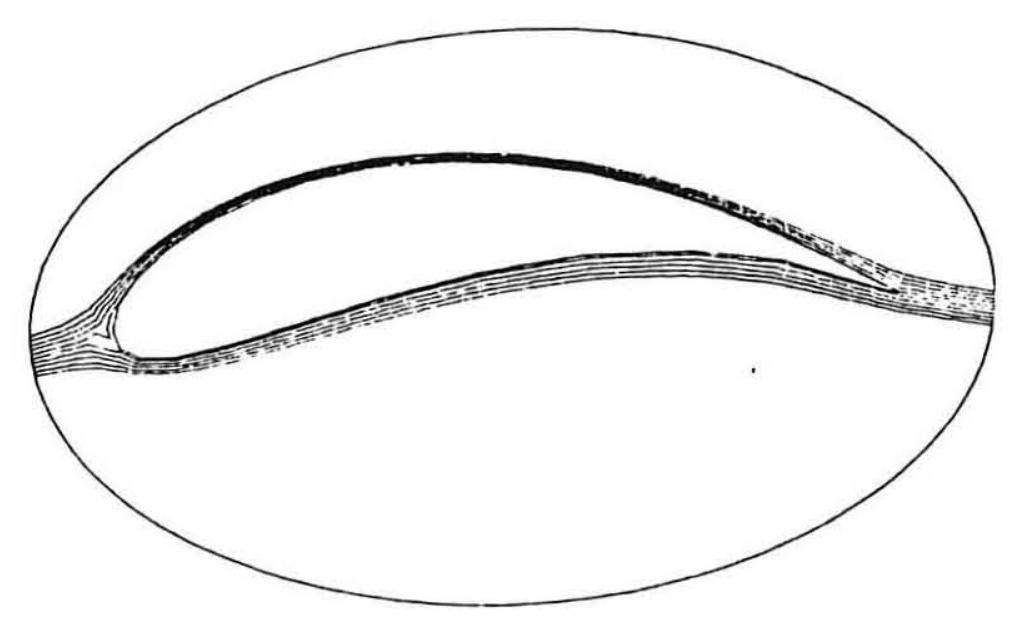

(b)

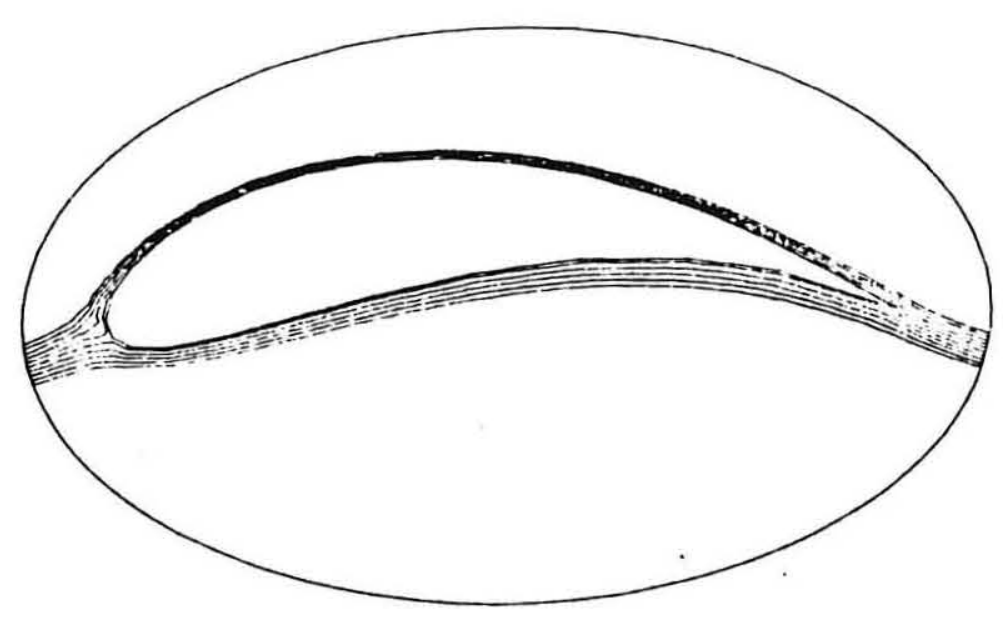

(c)

Fig. 3. Uniform flow over an airfoil; (a) correct circulation $\Gamma=0.6$,

(b) $\Gamma=0.5$

(c) $r=0.7$

6 
Table 1.

\begin{tabular}{|c|c|c|c|c|c|c|c|c|}
\hline \multirow[t]{3}{*}{$Y=0.0$} & $x$ & -2.25 & -2.15 & -0.95 & -0.35 & 1.05 & 2.15 & 2.25 \\
\hline & Theoretical & 0.619 & 0.426 & 0.786 & 0.705 & 0.647 & 0.893 & 0.909 \\
\hline & Numerical & 0.610 & 0.425 & 0.787 & 0.707 & 0.647 & 0.893 & 0.908 \\
\hline \multirow[t]{3}{*}{$x=0.05$} & $y$ & -1.5 & -1.0 & -0.5 & 0.0 & 1.0 & 1.5 & \\
\hline & Theoretical & 0.802 & 0.770 & 0.728 & 0.674 & 1.615 & 1.460 & \\
\hline & Numerical & 0.802 & 0.770 & 0.729 & 0.676 & 1.615 & 1.459 & \\
\hline
\end{tabular}

These results were computed using a mesh density of $\sim 850$ points over an ellipse with a major axis of 2.5 and a minor axis of 1.5 .

\section{Uniform Field Over an Iron Ring}

Suppose we place an iron ring, with inner and outer radius a, b respectively, in a uniform external field, $B_{\text {ext }}$. One can show that for constant permeability $\mu$, the ratio of the field in the ring $B_{\text {int }}$, to the external field $B_{\text {ext }}$ can be written as:

$$
\frac{B_{\text {int }}}{B_{\text {ext }}}=\frac{4 \mu b^{2}}{\left(\mu^{2}+1\right)\left(b^{2}-a^{2}\right)+2 \mu\left(b^{2}+a^{2}\right)}
$$

For this example we have taken $a=0.7, b=1.1$, and $\mu=10$, and applied $B_{\text {ext }}$ $=1000$. The theoretical value of 453.52 should be compared with 453.86 calculated by FOIL (flux lines are plotted in Fig. 4a). Fig. 4b is a similar plot with an external field of $B_{\text {ext }}=10000.0$ and a realistic field-dependent permeability for the iron. In this case, the inner field, which is not a perfect dipole, varies from 6800 to 7800 Gauss. 

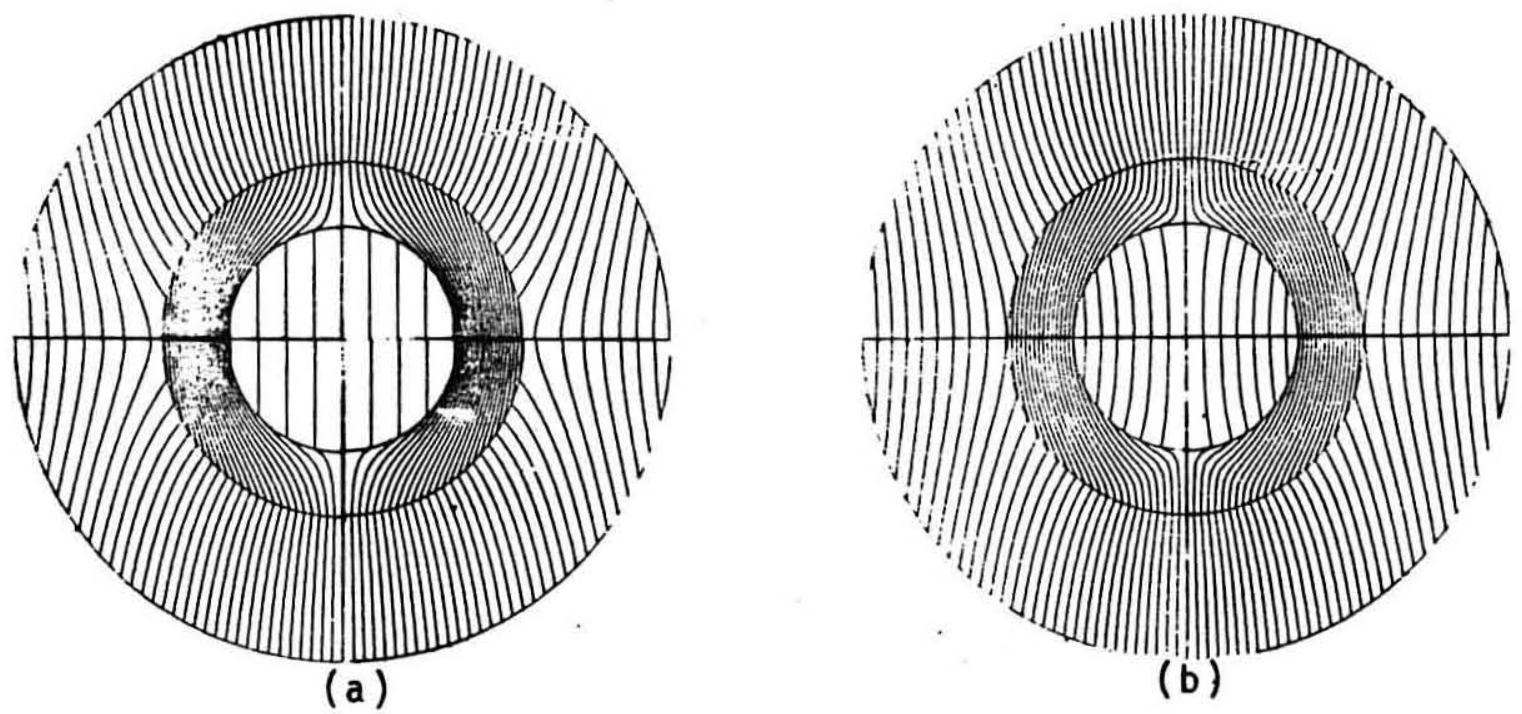

(b)

Fig. 4. Uniform field over an iron ring; (a) $\mu=10$, (b) $\mu=$ realistic field-dependent.

\section{REFERENCES}

1. S. Caspi, M. Helm, and L. J. Laslett, "Incorporation of a Circular Boundary Condition into the Program POISSON," LBID-887, Lawrence Berkeley Laboratory, February, 1984.

2. S. Caspi, M. Helm, and L. J. Laslett, "The Generalization of the Circular Boundary Condition into the Program POISSON to Include No Symmetry and Axis-Symmetry of Revolution," LBL-18063, Lawrence Berkeley Laboratory, July, 1984.

3. S. Caspi, M. Helm, and L. J. Laslett, "Incorporation of an Elliptical Boundary Condition into the Program POISSON," LBL-18798, Lawrence Berkeley Laboratory, December, 1984. 

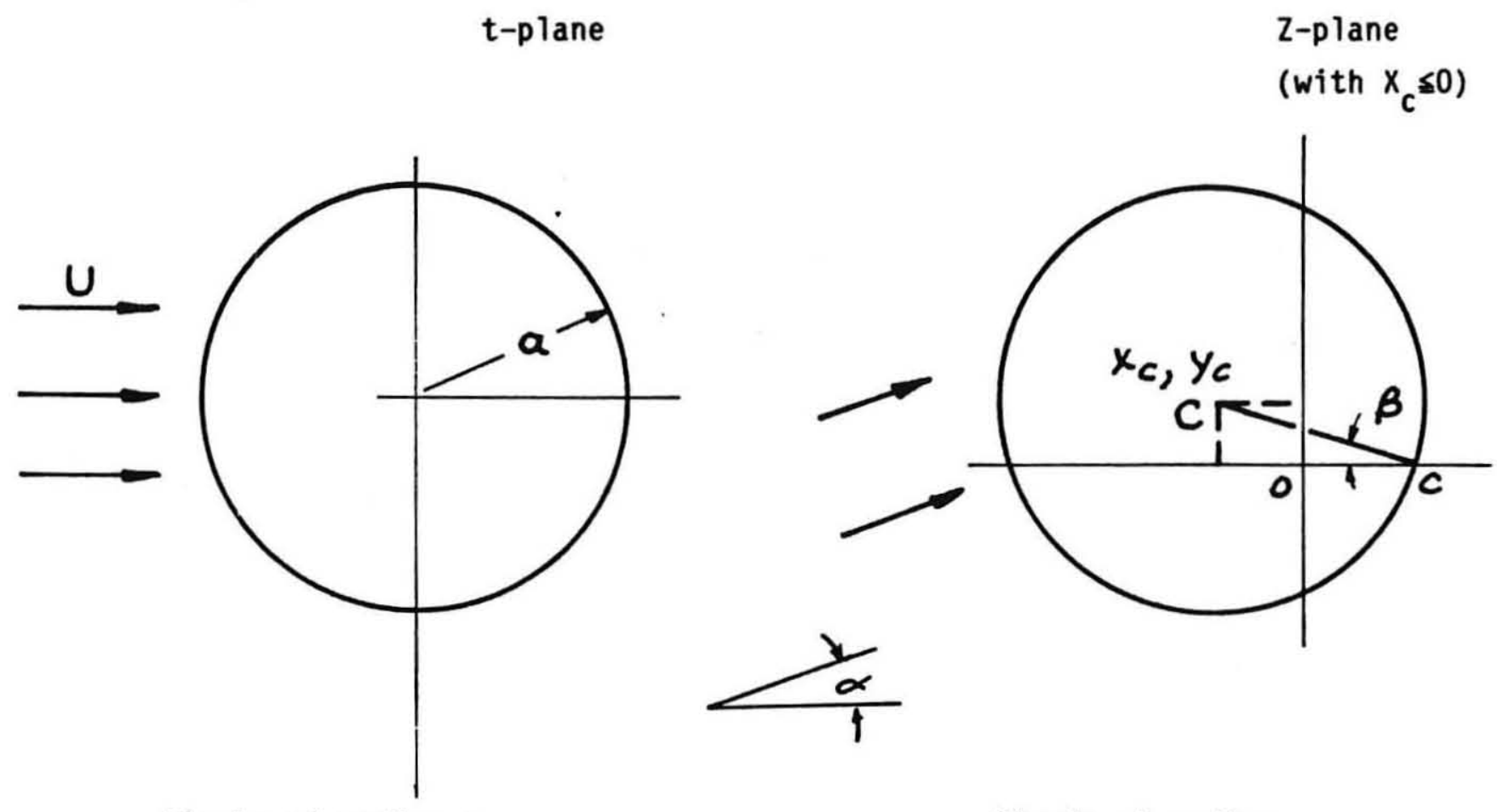

Circle of radius a

Circle of radius a

and center at $t=0$.

and center at $C$.

Polar coord for $t$

Origin of $z$ at 0

given by

$t=r e^{i \theta}$

Circle passes through

$=r(\cos \theta+i \sin \theta)$.

with $r=a$ on the circle.

$$
Z=c(\text { Real). }
$$

$\sin \beta=\frac{Y_{c}}{a}$.

$$
t=(z-\Delta) e^{-i \alpha}
$$

where

$$
\Delta=x_{c}+i y_{c} \text { with } x_{c} \leq 0 \text {. }
$$


In the $t$-plane, write

$$
W=\Phi+i A=U\left(t+\frac{a^{2}}{t}\right)+i r \ln t
$$

so that

$$
\begin{aligned}
\Phi & =\operatorname{Re}\left[U\left(t+\frac{a^{2}}{t}\right)+i r \ln t\right] \\
& =U\left(r+\frac{a^{2}}{r}\right) \cos \theta-r \theta \\
& =U\left(1+\frac{a^{2}}{x^{2}+y^{2}}\right) x-r \tan ^{-1} \frac{y}{x}
\end{aligned}
$$

and

$$
\begin{aligned}
A & =\operatorname{Im}\left[U\left(t+\frac{a^{2}}{t}\right)+i \Gamma \ln t\right] \\
& =U\left(r-\frac{a^{2}}{r}\right) \sin \theta+r \ln r \\
& =U\left(1-\frac{a^{2}}{x^{2}+y^{2}}\right) y+\frac{r}{2} \ln \left(x^{2}+y^{2}\right),
\end{aligned}
$$

expressions that satisfy the Riemann-Cauchy relations

$$
\frac{\partial \Phi}{\partial x}=\frac{\partial A}{\partial y} \quad \text { and } \quad \frac{\partial \Phi}{\partial y}=-\frac{\partial A}{\partial x}
$$

for an analytic function $\Phi+$ iA of a complex argument $x+i y$.

The velocity components in the t-plane can be obtained

$$
\begin{aligned}
& \text { as } \underset{t \text {-plane }}{\vec{v}}=\hat{e}_{x} \frac{\partial A}{\partial y}-\hat{e}_{y} \frac{\partial A}{\partial x}=\left[u\left(1-a^{2} \frac{x^{2}-y^{2}}{\left(x^{2}+y^{2}\right)^{2}}\right)+\Gamma \frac{y}{x^{2}+y^{2}}\right] \hat{e}_{x} \\
& -\left[2 U a^{2} \frac{x y}{\left(x^{2}+y^{2}\right)^{2}}+r \frac{x}{x^{2}+y^{2}}\right] \hat{e}_{y} \\
& =\hat{e}_{r} \frac{1}{r} \frac{\partial A}{\partial \theta}-\hat{e}_{\theta} \frac{\partial A}{\partial r}=\left[U\left(1-\frac{a^{2}}{r^{2}}\right) \cos \theta\right] \hat{e}_{r} \text {. } \\
& -\left[U\left(1+\frac{a^{2}}{r^{2}}\right) \sin \theta+\frac{r}{r}\right] \hat{e}_{\theta} \text {. }
\end{aligned}
$$


The magnitude of this velocity in the $t$-plane may be obtained from these components, or directly as

$$
\begin{aligned}
|\vec{v}|_{t-p l a n e} & \left|\frac{d w}{d t}\right|=\left|U\left(1-\frac{a^{2}}{t^{2}}\right)+i \frac{\Gamma}{t}\right| \\
& =\left|U\left(1-\frac{a^{2}}{r^{2}} \cos 2 \theta+i \frac{a^{2}}{r^{2}} \sin 2 \theta\right)+i \frac{\Gamma}{r} \cos \theta+\frac{\Gamma}{r} \sin \theta\right| \\
& =\sqrt{\left[U\left(1-\frac{a^{2}}{r^{2}} \cos 2 \theta\right)+\frac{\Gamma}{r} \sin \theta\right]^{2}+\left[U \frac{a^{2}}{r^{2}} \sin 2 \theta+\frac{\Gamma}{r} \cos \theta\right]^{2}} \\
& =\sqrt{U\left(1-2 \frac{a^{2}}{r^{2}} \cos 2 \theta+\frac{a^{4}}{r^{4}}\right)+\frac{2 U \Gamma}{r}\left(1+\frac{a^{2}}{r^{2}}\right) \sin \theta+\frac{\Gamma^{2}}{r^{2}}}
\end{aligned}
$$

At the surface of the circle -- i.e., at $r=a$-- this velocity in the t-plane becomes

$$
\begin{aligned}
& \vec{v} \underbrace{}_{t-p \text { lane }} \\
& \text { at } r=a
\end{aligned}
$$

Stagnation points occur on this surface, at angles $\theta_{S}$, if $\Gamma=-2 a U$ sin $\theta_{S}$ (for $\Gamma \leqq 2 \mathrm{aU})$; a single such point occurs, at $\theta_{s}=-\frac{\pi}{2}$ radian, for $\Gamma=2 \mathrm{aU}$.

The complex position variables for the $t$ and $z$ planes are related by

$$
t=(z-\Delta) e^{-i \alpha} \text {, }
$$

where $\Delta=x_{c}+i y_{c}$ with $x_{c} \leq 0$.

$$
\text { . Note: }\left|\frac{d t}{d z}\right|=1 \text {. }
$$

In anticipation of performing a Joukowski transformation, we wish that $v_{t-p l a n e}$
$t-0$ at $Z$ = (a point on the surface of the circle). That is: we wish $v=0$ at $t=\left[\left(c-x_{c}\right)-i Y_{c}\right] e^{-i \alpha}$, which is a point situated 
on the circle in the t-plane at a polar coordinate angle $\theta=-\tan ^{-1}\left(\frac{Y_{c}}{c-X_{c}}-\alpha\right)=-(\beta+\alpha)$.

To achieve the result $V=0$ (stagnation) at this point we therefore adopt the value $\Gamma=2 \mathrm{aU} \sin (B+\alpha)$.

[We note, in passing, that this value of $r$ would vanish (and hence the presumed lift would vanish) if $\alpha=-B$.

The Joukowski transformation that may be employed to generate, by transformation from a circle, an "airfoil" in the $\zeta=\xi+$ in is

$$
\zeta=z+\frac{c^{2}}{z} \quad \text { [Complex arithmetic] }
$$

For any point $\zeta$ in this $\zeta$-plane, we may solve for the corresponding value of Z:

$$
z=\frac{\zeta}{2}+\sqrt{\left(\frac{\zeta}{2}\right)^{2}-c^{2}}
$$

(selecting the sign of the square root so that points outside the airfoil fall outside the circle in the $Z$ plane),

and

$$
t=(z-\Delta) e^{-i \alpha} \text {. }
$$

To compute the magnitude of velocity (at points on the surface of, or exterior to, the airfoil) we then may write

$$
\begin{aligned}
&|v|\left.=\left|\frac{d W}{d \zeta}\right|=\left|\frac{d W / d t}{d \zeta / d z}\right| \quad \text { (since }\left|\frac{d t}{d z}\right|=1\right) \\
&=\left|\frac{U\left(1-\frac{a^{2}}{t^{2}}\right)+i \frac{r}{t} \mid}{\left(1-\frac{c}{z}\right)\left(1+\frac{c}{z}\right)}\right| \\
& \text { [Complex arithmetic] }
\end{aligned}
$$

This quotient should be readily computed (say by use of complex arithmetic) save for the point at the trailing edge of the airfoil (where the value of $z$ is c) and an indeterminate form occurs. 


\section{APPENDIX B}

The program AFOIL (Library RUTH) is an interactive program designed to tabulate and/or plot the results of transforming a circle (radius "a") in the $Z=X+i Y$ plane into the $\zeta=\xi+$ in plane by means of the transformation

$$
\zeta=z+\frac{c^{2}}{z}
$$

(wherein we treat $c$ as real).

If a point on the circle coincides with $z= \pm c+i 0$, a singularity (vanishing derivative $d \zeta / d z$ ) occurs. A Joukowski airfoil results if the circle intersects one such point (to provide a sharp trailing edge), but surrounds the other such point. The vertical displacement of the center of the circle then gives the curving of the airfoil, while the horizontal displacement (say to the left) contributes to the thickness.

In using the program, one firsts types the value of the parameter c (e.g., $c=1$ ). The value of the circle radius "a" may be entered if desired, followed by the $X$ and $Y$ coordinates of the center of the circle. Alternatively, the program will so compute the radius "a" so that the circle will pass through one of the points $\pm c+i 0$ (and will surround the other).

The angle $B$ locates the line from $c$ to the center of the circle with respect to the real axis in the Z-plane, as illustrated. 


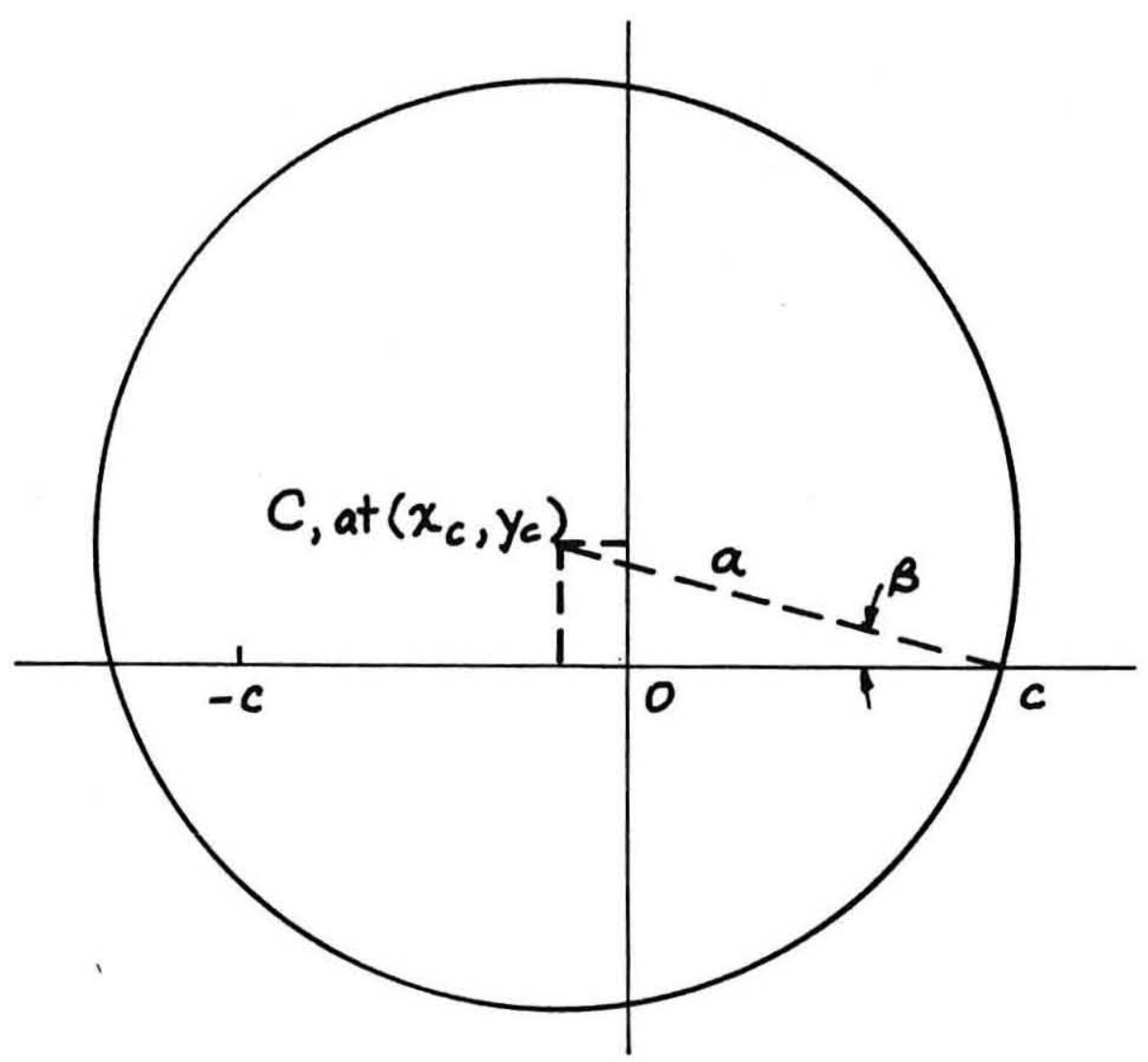

$\underline{\text { Z-Plane }}$

0: Orgin of Z-plane

C: Center of Circle

The sketch suggests an example in which, with $c=1$, the circle has been arranged to pass through the point $+c+i 0$. With

$$
X_{c}=-0.15 \text { and } Y_{c}=+0.30
$$

this requires that

$$
\begin{aligned}
& a=\sqrt{1.4125} \cong 1.18848643 \\
& B=\tan ^{-1} \frac{0.30}{1.15} \cong \tan ^{-1} 0.260869565
\end{aligned}
$$

and

$\cong 14.620874$ degrees.

while the point $-c+i 0$ remains within the circle.

The result of the transformation $\zeta=z+\frac{c^{2}}{z}$ applied to this circle is shown in the $\zeta$ plane on the following figure, whereon the axes extend between the limits $\pm 4 \mathrm{c}$; i.e., between the limits \pm 4.0 in the present case. [The "trailing" edge shown here at $\zeta=2.0, n=0$ is the result of the transformation applied to the point $X=c=1, Y=0$.] 


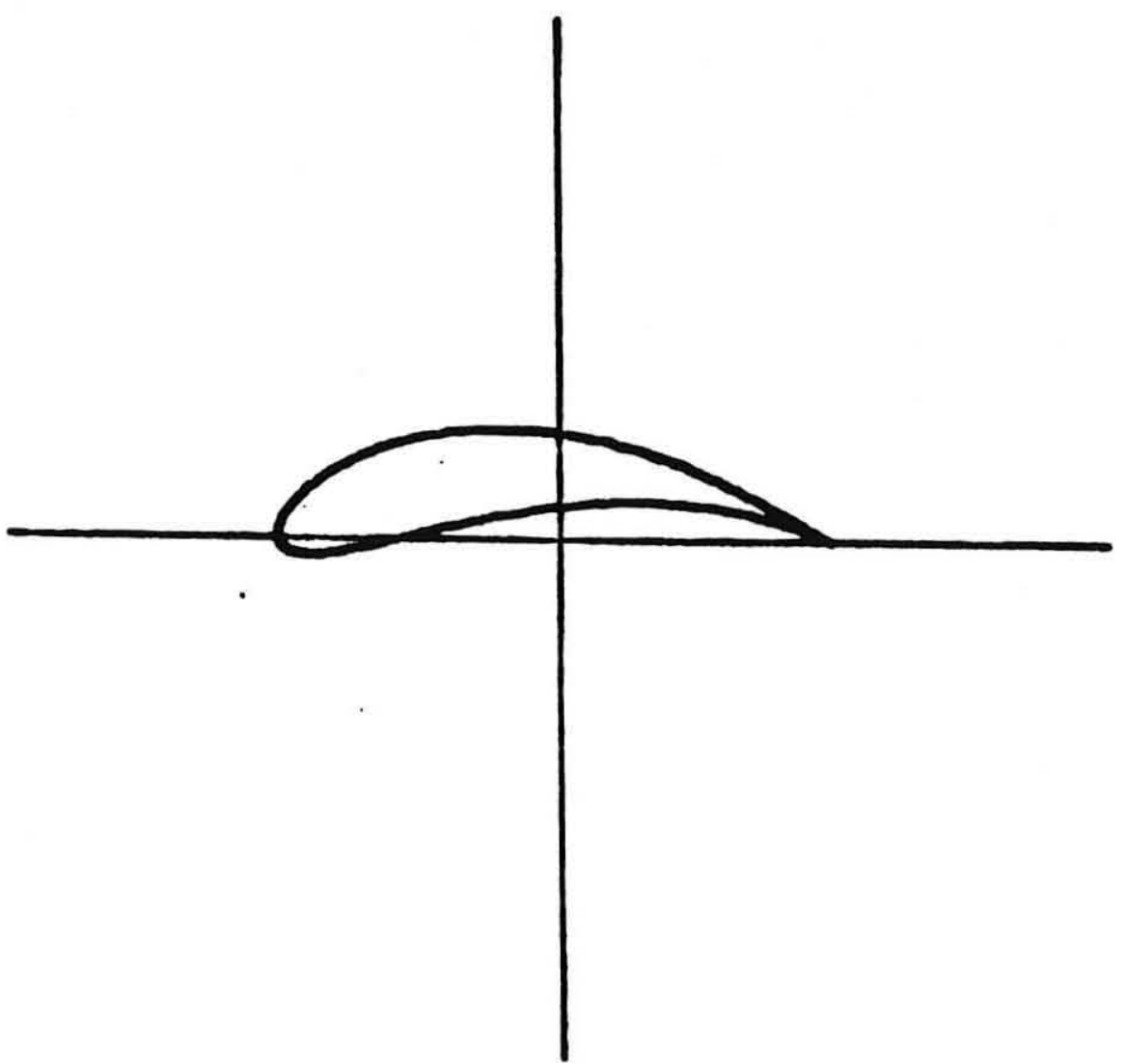

The transformation results from

$$
\begin{aligned}
& X=a \cos \theta+X_{c}=a \cos \theta-0.15 \\
& Y=a \sin \theta+Y_{c}=a \sin \theta+0.30
\end{aligned}
$$

$$
\text { with } a=\sqrt{1.4125} \cong 1.18848643 \text {, using } \zeta=Z+c^{2} / Z \text { with } c=1 \text {. }
$$

Intersections with the horizontal axis $n=0$ occur at:

$\theta$ (deg.)

$-14.620874(=-B)$

$194.620874(=180+B)$

247.750976 $x$

1.0
-1.3
-0.6
Y

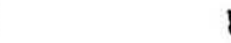

$\boldsymbol{\xi}$

For the maximum extension to the left in the $\zeta$-plane, we have approximately:
$\theta \cong 190.2 \mathrm{deg}$.
$x \cong-1.32$
$Y \cong 0.09$
$\xi \cong-2.074$
$n \cong 0.038$
$\cong 0.04$ 